\title{
Preliminary Step Towards Wire-Driven Parallel Suspension Systems for Static and Dynamic Derivatives of the Aircraft in Low-Speed Wind Tunnels
}

\author{
Yaqing Zhenga,b, Qi Lina ${ }^{*}$, Xiongwei Liuc and Peter Mitrouchev ${ }^{d}$ \\ $a^{*}$ Department of Aeronautics, Xiamen University, Xiamen,361005,China \\ ${ }^{b}$ College of Mechanical Engineering and Automation, Huaqiao University, Quanzhou, 362021, China \\ c School of Computing, Engineering and Physical Sciences, University of Central Lancashire, Preston, UK \\ ${ }^{d}$ G-SCOP Laboratory, GrenobleINP-UJF, FRE 3028 CNRS, 46 Avenue Félix Viallet, 38031 Grenoble Cedex1, France
}

Received 5 July 2008; accepted 7 June 2009

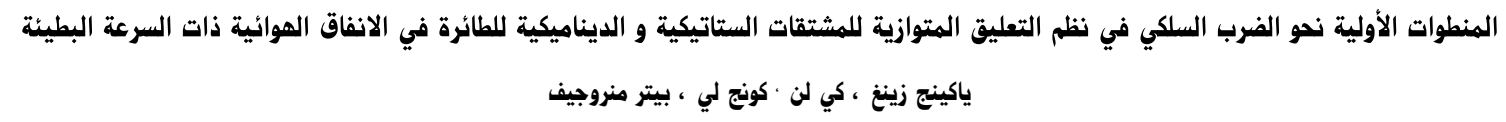

الهزردات الهثتاهية: نفق هوائي ، نظام تعليق ، الدفع السلكي ، مشتقات ستاتيكية و داينمكية.

\begin{abstract}
The wind tunnel test is one important way to obtain the aerodynamic derivatives of aircrafts. These derivatives are necessary when the guidance and control systems of the aircraft are designed and when the dynamic quality of the aircraft is analyzed as well. The results of experiments of the static derivatives and dynamic derivatives of the aircraft in low-speed wind tunnels have revealed that there are some unavoidable drawbacks such as the interference of the streamline flow brought about by the strut in the traditional strut suspension system. A cable-mounted system is very suitable for experiments of the static derivatives of an aircraft, but it cannot be used in the experiments of dynamic derivatives. In order to use the same wire-driven parallel suspension system to realize the static and dynamic derivates experiments in low-speed wind tunnels, a survey of the research work addressed within the Wire-Driven Parallel Suspension Systems (WDPSS-8) project is presented in this paper. The results show that WDPSS-8 can be successfully used in experiments of static derivatives, and that it has potentiality to be used in experiments of dynamic derivatives. In the issues in the theoretical aspect the issues have been handled. However, much work should be done in the experimental aspects. The research outcomes of WDPSS-8 will help the Chinese set up Chinese brands in the field of wind tunnel tests of aircrafts.
\end{abstract}

Keywords: Wind tunnel, Suspension system, Wire-driven, Static and dynamic derivatives

\section{Introduction}

The data on aerodynamic characteristics are when the guidance system and the control system of the aircraft are

*Corresponding author’s email: qilin@xmu.edu.cn designed. These data are essential when the dynamic behavior of the aircraft is analyzed, indicating the great importance attached to the stability and maneuverability of modern aircraft. The dynamic aero characteristics of 
the aircraft are closely related with the aerodynamic forces and torques exerted on the aircraft. In analyzing the aerodynamic characteristics, these aerodynamic forces and torques can be expressed by some aerodynamic coefficients and derivatives. The latter are the derivatives of the aerodynamic coefficients to some parameters such as angle of attack, angle of side-slip and Mach number which are called static derivatives. The derivatives of the aerodynamic coefficients to some parameters such as pitching angular velocity, rotating angular velocity and yawing angular velocity are called dynamic derivatives (Sun, 1999).

The wind tunnel test is one important way to obtain the aerodynamic derivatives of the aircraft. During a windtunnel tests it is necessary to place the scale model of the aircraft in the streamline flow of the experimental section of the wind tunnel using some kind of suspension system. The suspension system will have a lot of influences on the reliability of the results of the wind tunnel tests. The traditional rigid suspension systems have some unavoidable drawbacks for the experiments of static and dynamic derivatives such as the serious interference of the strut on the streamline flow (Zhang et al. 2006; Roas, 2001; Bernhardt and Williams, 2000; Cao and Deng, 1998 and $\mathrm{Du}$ and Wang, 2003). The cable-mounted systems for wind tunnel tests, developed in the past several decades, deal with the contradiction between the supporting stiffness and the interference on the streamline flow (Bian et al. 1999; Shen and Huang, 1999; Bennett et al. 1978; Liu et al. 2001, Wang et al. 2004 and Griffin 1991). However their mechanism is not robotic and consequently quite different from wire-driven parallel suspension systems in attitude-control schemes and force-measuring principle. In addition they can not be used in dynamic derivatives experiments (Graffin, 1992). Instead, the freeflight simulation concept in wind tunnels through an active suspension such as six-DOF (degrees-of-freedom) Wire-Driven Parallel Suspension Systems (WDPSS), coming from the research improvement in wire-driven parallel manipulator and force control, is suitable for calculating the aerodynamic derivatives of the aircrafts Lafourcade et al. 2002; onera.fr/desd/sacso/index.php; Liu, 2004 and Zheng 2004). The static derivatives can be obtained by researching the static models without winds and with winds (Zheng, 2006). Some successful achievements in this field have been made in the Suspension Active pour Soufflerie (SACSO) project for nearly 8 years and it has been applied in vertical wind tunnel tests with a wind speed of $35 \mathrm{~m} / \mathrm{s}$ for fighters at the first stage of their conceptual design. However the system can not be used in experiments of dynamic derivatives in low-speed wind tunnels (Lafourcade et al. ).

In this context our goal is to introduce some contributions in the field of wire-driven parallel suspension systems for static and dynamic derivatives of the aircraft in low-speed wind tunnels. Under the sponsorship of NSFC (National Natural Science Foundation of China), the authors and the co-authors have done over 6-years of deep and systematic research work on a 6-DOF wiredriven parallel suspension system with 8 wires (WDPSS8) for low-speed wind tunnels deep and systematic research (Liu et al. 2004; Zheng, 2004; Zheng et al. 2005, Zheng et al. 2007, Lin et al. 2006; Zheng, 2006; Liu et al. 2006; Zheng, 2006 and Liang et al. 2007). The attitude control and aerodynamic coefficients (static derivatives) of the scale model have been investigated in theory and in experiment. A prototype (WDPSS-8) has been realized (built) and tested in an open return circuit low speed wind tunnel with a wind speed of $30 \mathrm{~m} / \mathrm{s}$. The experimental section of the wind tunnel is rectangular, with a length of 1 meter, a width of 0.52 meters and a height of 0.42 meters (Liu et al. 2006). The authors also found the possibility of using the prototype (WDPSS-8) for the experiment of dynamic derivatives by successfully implementing the single-DOF oscillation control of the scale model (Liang et al. 2007; Hu, 2008 and Lin et al. 2008).

Concerning the possibility of using the same wire-driven parallel suspension system to realize the static and dynamic derivates experiments in low-speed wind tunnels, a survey of the research work finished on some key issues of WDPSS-8 for the experiments of the static and dynamic derivatives will be addressed. The research results of the experiments, especially those of the experiments of the dynamic derivatives, will provide some criterion of experimental data for free flight and some effective experimental methods about the controllability capability of post-stall maneuvers in the design of Large Aircraft and new generation of Military Aircraft. This will help the Chinese set up the Chinese Brands in the field of wind tunnel tests of aircraft.

The rest of the paper is organized as follows: The key issues of WDPSS-8 for the experiments of static derivatives of aircrafts in Low-Speed Wind Tunnels (LSWT) are given in the next Section 2. The key issues of WDPSS8 for the experiments of dynamic derivatives of aircraft in LSWT are presented in Section 3 by showing the possibility to use the same WDPSS-8 for static and dynamic derivates experiments in LSWT. Finally, discussions and future work are suggested in Section 4.

\section{WDPSS-8 for Experiments of Static Deri- vatives of Aircraft in Low-Speed Wind Tunnels}

\subsection{Two WDPSS-8 Prototypes}

\subsubsection{A Manually-operated WDPSS-8 Prototype Validated in a closed-Circuit Wind Tunnel}

A wire-driven parallel manipulator is a closed-loop mechanism where the moving platform is connected to the base via wires by a multitude of independent kinematic chains. The number of a moving platform's degree of freedoms (DOFs) is defined as the dimension of linearity space which is positively spanned by all the screws of the structure matrix of the manipulator. So the moving platform of a 6-DOF's completely or redundantly restrained 
wire-driven parallel manipulator is driven by at least 7 or more wires.

Meanwhile, a 6-DOF wire-driven parallel suspension system (WDPSS) is essential for the free fight of the aircraft in a 3-dimensional space wind tunnel. Fig. 1(a) shows the concept of a 6-DOF WDPSS driven by 8 wires (WDPSS-8), the geometric definition of which is shown in Fig. 1(b) and the geometric parameters of which are listed in Table 1. A manually-operated prototype of such a design shown in Fig. 1 (c) is built and tested in a closedcircuit LSWT. To implement the scenario for the attitude adjustment of the aircraft, a driving mechanism adjusted manually has been developed which allows the aircraft model to maneuver, ie. to permit roll, pitch and yaw motion. For the WDPSS-8, each cable will be attached to a driving unit, which consists of a screw bar and a driving nut, as shown in Fig.1(d). A commercial load cell interfaced to the cable shown in Fig. 1(e) is used to measure the tension of a cable. To avoid extra interference, the strain gage balance and driving unit are attached to the wind tunnel frame on the outside of the tunnel, as shown in Fig. 1(c) and (d).However, Fig. 1(f) shows the aircraft model mounted on a conventional strut suspension system in the same LSWT.

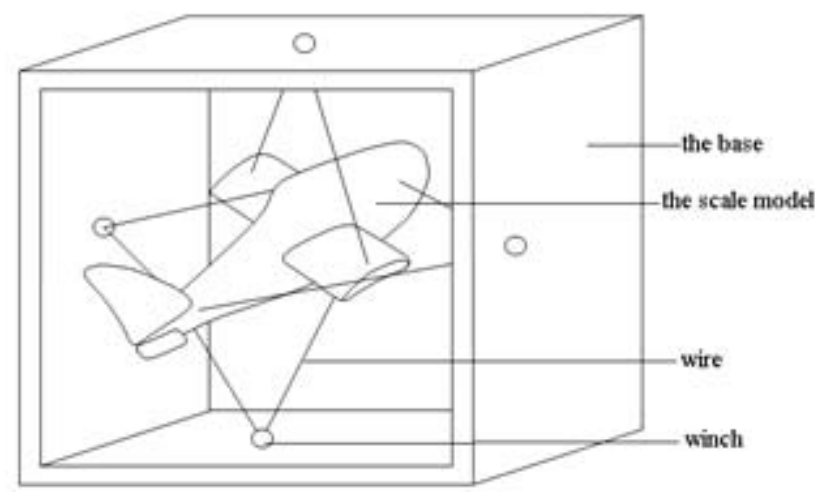

(a) The concept of wire-driven parallel suspension system with 8 wires (WDPSS-8).

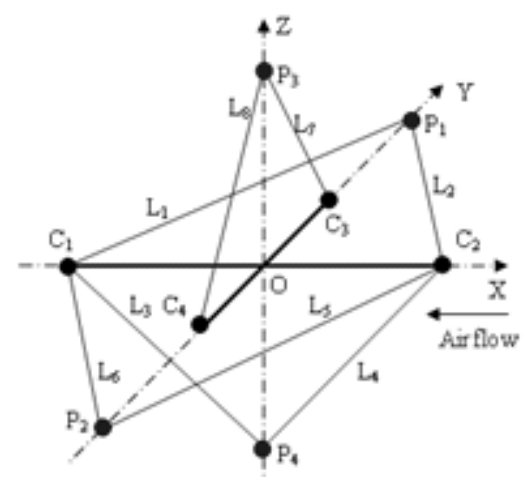

(b) Geometric definition of the WDPSS-8 prototype.

The WDPSS-8 prototype has been validated by windtunnel tests in a wind speed of $28.8 \mathrm{~m} / \mathrm{s}$ and it has been

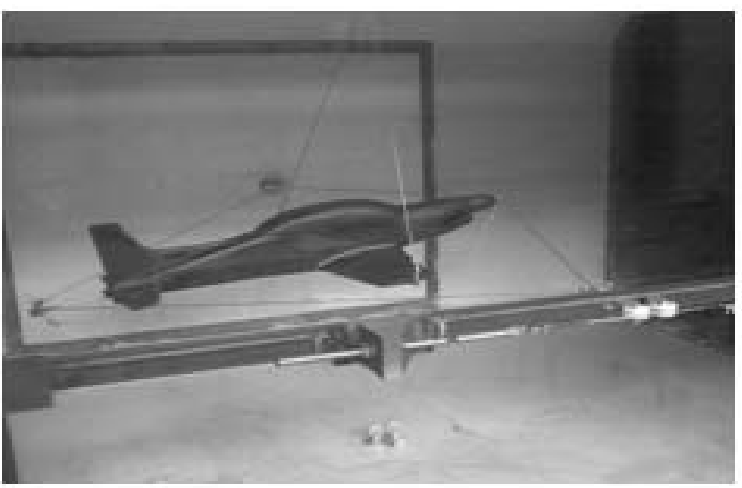

(c) WDPSS-8 tested in LSWT in a wind speed of $28.8 \mathrm{~m} / \mathrm{s}$.

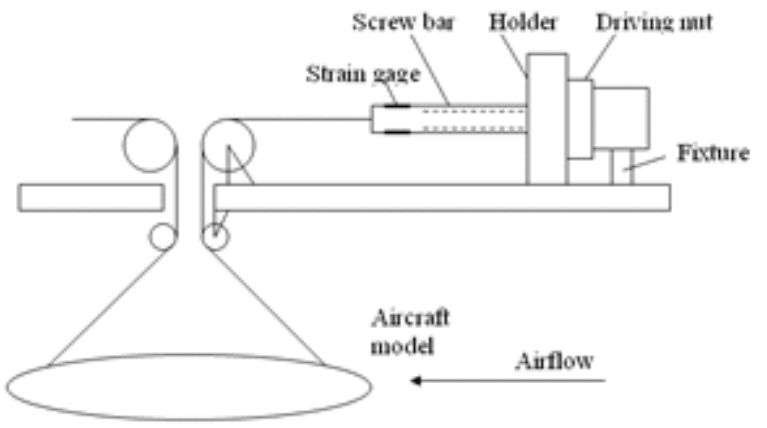

(d) Driving unit of WDPSS-8.

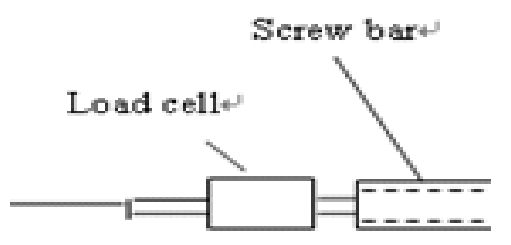

(e) Load cell interface.

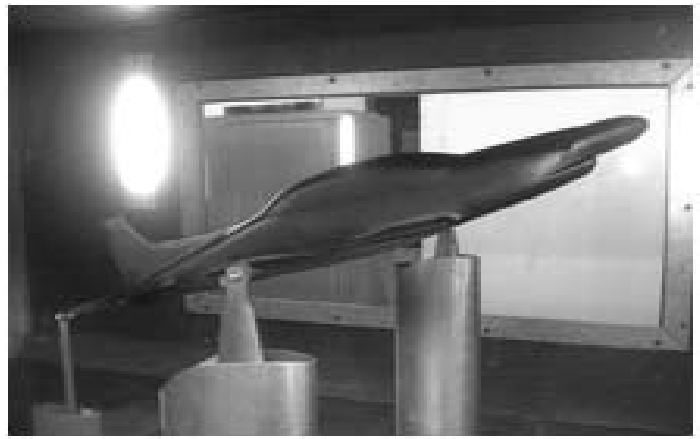

(f) Strut suspension system.

Figure 1. Comparison of 2 different suspension systems.

found in the experiments that there is little vibration occurring at the end of the scale model which is less than that in the corresponding traditional strut suspension system shown in Fig. 1 (f), from which it has been found that the fundamental frequency of WDPPS-8 is smaller than that of the corresponding traditional strut suspension. 
Table 1. Geometric parameters of the WDPSS-8 prototype (unit in $\mathbf{m m}$ )

$$
\begin{array}{ccccc}
\text { Pitch }\left(^{\circ}\right) & \mathbf{C}_{\mathbf{1}}(\mathbf{X}, \mathbf{Y}, \mathbf{Z}) & \mathbf{C}_{2}(\mathbf{X}, \mathbf{Y}, \mathbf{Z}) & \mathbf{C}_{3}(\mathbf{X}, \mathbf{Y}, \mathbf{Z}) & \mathbf{C}_{4}(\mathbf{X}, \mathbf{Y}, \mathbf{Z}) \\
-6 & -300,0,-32 & 300,0,32 & 0,605,-30 & 0,-605,-30 \\
0 & -302,0,0 & 302,0,0 & 0,605,-30 & 0,-605,-30 \\
6 & -300,0,32 & 300,0,-32 & 0,605,-30 & 0,-605,-30 \\
12 & -295,0,63 & 295,0,-63 & 0,605,-30 & 0,-605,-30 \\
& \mathrm{P}_{1}(\mathrm{X}, \mathrm{Y}, \mathrm{Z}) & \mathrm{P}_{2}(\mathrm{X}, \mathrm{Y}, \mathrm{Z}) & \mathrm{P}_{3}(\mathrm{X}, \mathrm{Y}, \mathrm{Z}) & \mathrm{P}_{4}(\mathrm{X}, \mathrm{Y}, \mathrm{Z}) \\
& 0,0,420 & 0,0,-420 & 0,605,0 & 0,-605,0
\end{array}
$$

2.1.2 Another WDPSS-8 Prototype tested in an open return-Circuit Wind Tunnel

Figure 2(a) shows another geometric definition of WDPSS-8 and its the structural parameters are listed in Table 2.

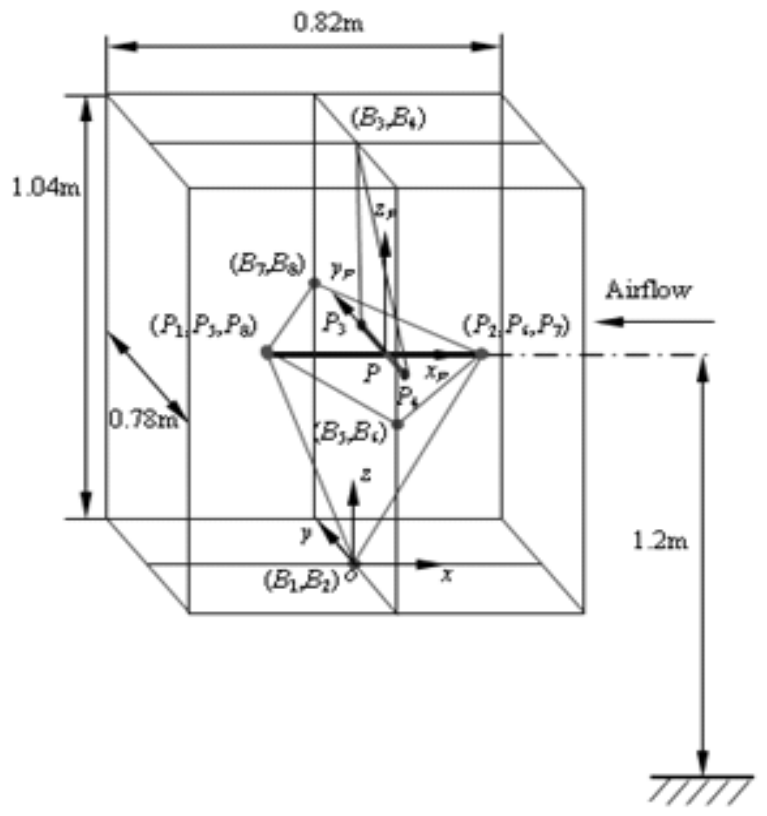

(a) Another geometric definition of WEPSS-8 prototype.

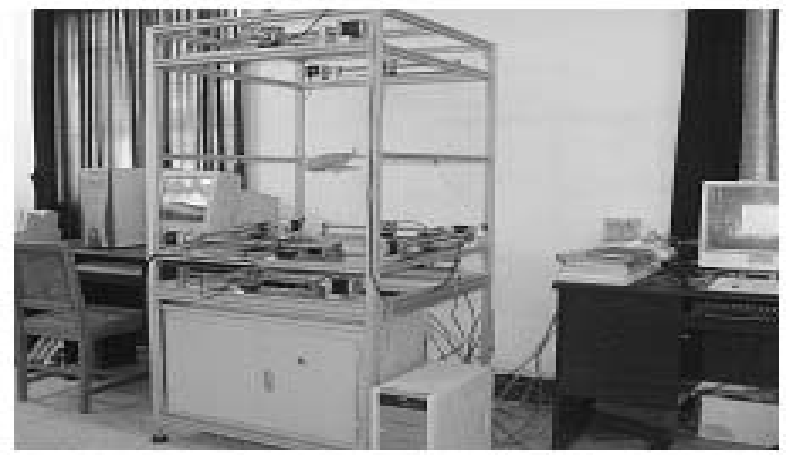

(b) Prototype of WDPSS-8

Figure 2. Another WDPSS-8 prototype tested in an open return-circuit wind tunnel.
Table 2. Structural parameters of the WDPSS-8 prototype (unit in mm)

$$
\begin{array}{cccc}
\mathbf{P}_{1}\left(x_{P}, y_{P}, \mathbf{Z}_{P}\right) & \mathbf{P}_{2}\left(x_{P}, y_{P}, \mathbf{Z}_{P}\right) & \mathbf{P}_{3}\left(x_{P}, y_{P}, \mathbf{Z}_{P}\right) & \mathbf{P}_{4}\left(x_{P}, y_{P}, \mathbf{Z}_{P}\right) \\
-120,0,0 & 80,0,0 & 0,50,0 & 0,-50,0 \\
\mathrm{~B}_{1}(\mathrm{X}, \mathrm{Y}, \mathrm{Z}) & \mathrm{B}_{3}(\mathrm{X}, \mathrm{Y}, \mathrm{Z}) & \mathrm{B}_{5}(\mathrm{X}, \mathrm{Y}, \mathrm{Z}) & \mathrm{B}_{7}(\mathrm{X}, \mathrm{Y}, \mathrm{Z}) \\
0,0,0 & 0,0,1040 & 0,-390,520 & 0,390,520
\end{array}
$$

A test for this WDPSS-8 for low-speed wind tunnels is also realized, shown in Fig. 2(b), in which the 3 rotational attitude control of the scale model (yaw, roll and pitch) has been accomplished. During the wind tunnel testing for the static derivatives, it is necessary to place the scale model using the suspension system in the streamline flow of the experimental section in wind tunnels, using the suspension system, and the attitude of the scale model should be adjustable. The inverse kinematics problem deals with the calculation of the length of each cable corresponding to the given different attitude of the scale model which will provide the data for the position control. The modeling of inverse pose kinematics of WDPSS-8 can be found in references (Zheng et al. 2005 and Zheng et al. 2006).

\subsection{Calculation of the Static Derivatives}

Because the scale model moves in a quasi-static way during the LSWT experiment for the static derivatives, it is reasonable to calculate the aerodynamic force and torque exerted on it using the difference of the force and torque exerted on the scale model between without wind and with wind. As a preliminary research, the assumption that all constraints are perfectly applied with no resistance in pulleys or other mechanisms such as point-shaped joints which are required to maintain the geometry of the wires at the base and the scale model is given for convenience. May be this is not really the case, but it is reasonable because the attitude of the scale model is controlled and adjusted in a quasi-static way so that the errors in the mechanism configuration between without wind and with wind could be easily limited to a range which can be neglected.

The static model of WDPSS- 8 without wind can be expressed by:

$$
\boldsymbol{J}^{\mathrm{T}} \boldsymbol{T}+\boldsymbol{F}_{\mathrm{G}}=\mathbf{0}
$$

Here, $T$ is a vector $\left(t_{1} \ldots t_{8}\right)$ with 8 components, 0 is a vector with 6 components, $J^{T}$ is the structural matrix of the manipulator, $F_{G}$ is the gravity vector with 6 components.

The static model of WDPSS-8 with wind can be expressed by:

$$
J^{T} T^{\prime}+F_{G}+F_{\mathrm{A}}=\theta
$$

Here, $F_{A}$ is the vector of aerodynamic force and torque with 6 components.

From Eqs. (1) and (2), we can find that the equation $F_{\mathrm{A}}=J^{T}(T-T)$ is satisfied. 
In order to calculate the static derivatives (related to $F_{A}$ ), we should measure the tension of all wires and the posture of the scale model when the scale model is position-controlled without wind and with wind. 2 different groups of experiment of static derivatives using WDPSS8 with 2 different mechanism configuration parameters have been finished in a closed circuit low-speed wind tunnel and an open return circuit low-speed wind tunnel respectively, which will be stated in the following in detail.

\subsubsection{Experiment of Static Derivatives using WDPSS-8 finished in a closed Low-Speed Wind Tunnel}

As shown in Fig. 1 and Fig. 4, the experiments of static derivatives using WDPSS-8 and using the conventional strut suspension system have been finished in a closedcircuit low-speed wind tunnel with a wind speed of 28.8 $\mathrm{m} / \mathrm{s}$. The attack angle (pitch) can be adjusted from 00 to 18 ${ }^{0}$. Fig. 4 and Fig. 5 show the measured Lift and Drag forces versus the pitch angles and the measured momentversus the pitch angles using the conventional wind tunnel testing and using WDPSS-8 wind tunnel testing respectively.

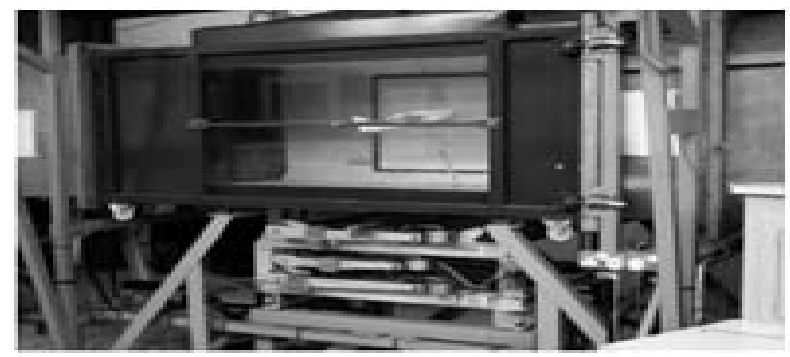

Figure 4. WDPSS-8 in a closed low-speed tunnel.

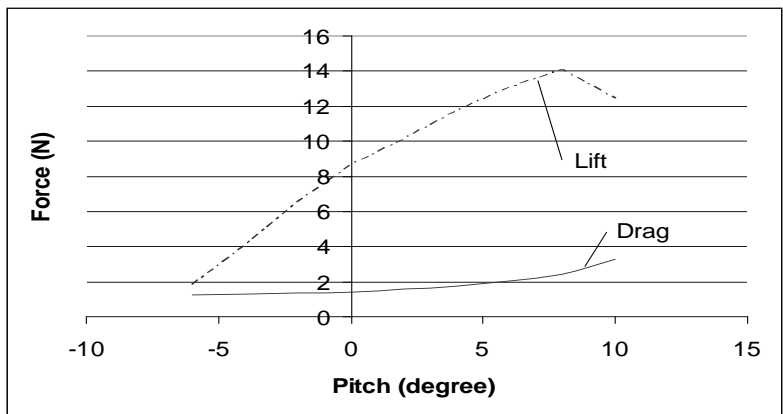

Figure 5(a) Measured lift and drag vs, pitch using the conventional wind tunnel testing.

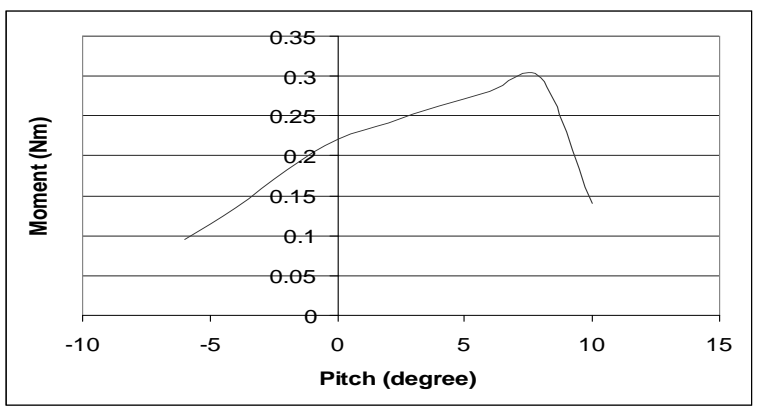

Figure 5(b) Measured moment vs. pitch using the conventional wind tunnel testing.

Figure 5. Conventional wind tunnel testing

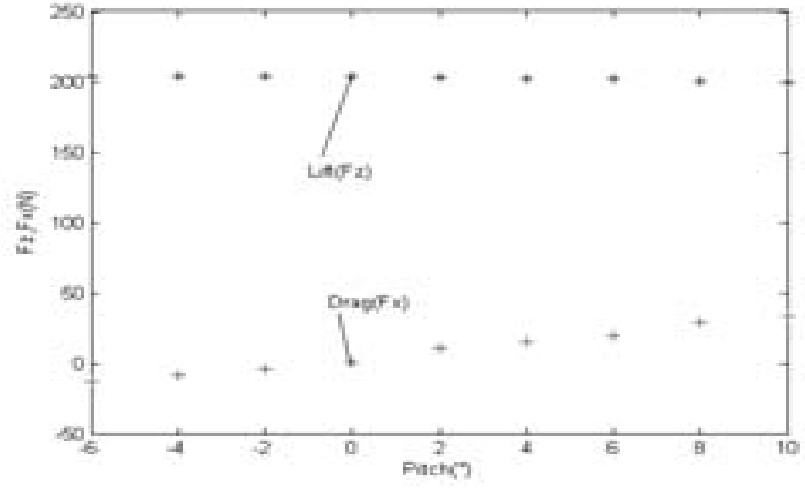

Figure 6(a) Measured lift and drag vs. pitch using WDPSS-8 wind tunnel testing.

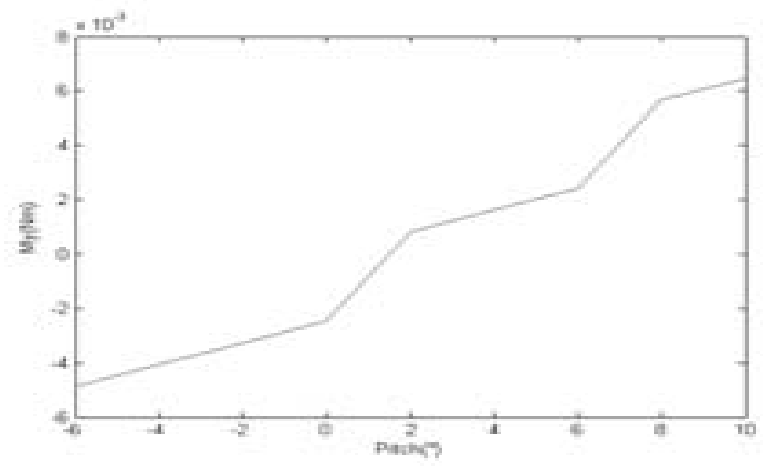

Figure 6(b) Measured moment vs. pitch using WDPSS-8 wind tunnel testing.

Figure 6. WDPSS-8 wind tunnel testing.

\subsubsection{Experiment of Static Derivatives using WDPSS-} 8 in Open Return Circuit Low Speed Wind Tunnel

With the experimental set-up in place, the wind tunnel testing by WDPSS-8 is run by inducing airspeed of $30 \mathrm{~m} / \mathrm{s}$, as shown in Fig. 7. For calculation of wire tension, the sensor NS-WL1, with a range of $10 \mathrm{~kg}$, was chosen.

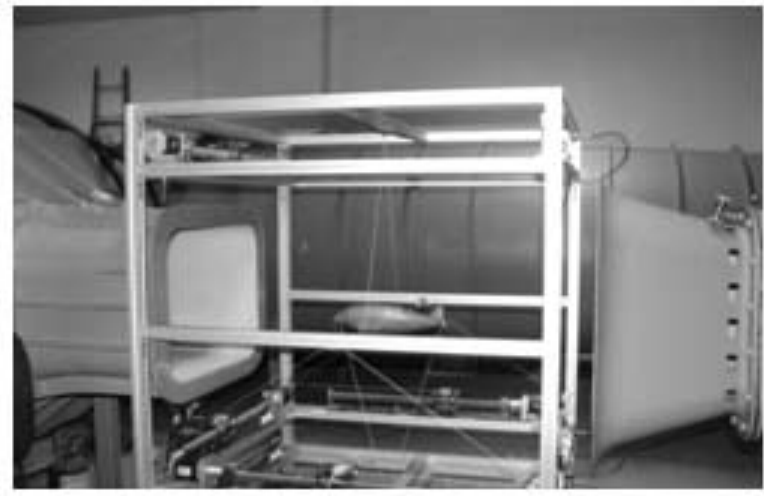

Figure 7. Wire-driven parallel suspension systems with 8 wires in the experiment of static derivatives.

A group of wind tunnel tests are done for the scale model of WDPPS-8 in an open return circuit low-speed wind tunnel and the experimental curves of lift coefficient versus angle of pitch, drag coefficient versus angle of pitch and lift/drag ratio versus angle of pitch are shown. 
As shown in Fig. 8, there are 3 experimental curves for wind tunnel testing of WDPSS-8 with a wind speed of $17.27 \mathrm{~m} / \mathrm{s}$. Though there are no data about the standard model as criteria, the curves are suitable for expressing the aerodynamic characteristics of aircraft.

\section{WDPSS-8 for the Experiments of Dynamic Derivatives of the Aircraft in Low-Speed Wind Tunnels}

In the test platform of WDPSS-8, the oscillation control of the scale model is controlled without wind and with wind respectively. The vibration displacement of the scale model is measured by the sensors inside the model such as micro gyro and micro accelerators. The aerodynamic force and torque are calculated by the dynamic equations of the systemm. Also the dynamic derivatives can be calculated by the real torques of motors without wind and with wind which can be measured by force sensors mounted on the axis of the motors. The following parts will state the issue in detail.

\subsection{Dynamic Modeling of the System and the Scheme of Oscillation Control}

The suspension system's dynamic modeling is necessary for the control system's design of the oscillation of the scale model. The following assumptions when carrying out the dynamic modeling are given as follows:

(1) The deformation of wires is so small that it can be neglected. The mass of the wires can be neglected as well.

(2) The dynamics of the actuators are neglected to simplify the dynamics model of the manipulator.

In references Zheng et al. 2007 and Zheng 2006, the total dynamic modeling of WDPSS-8 is written as:

$\left(M_{o}+J^{T} M J\right) X+\left(M_{o}+J^{T} M J+J^{T} B J\right) X=J^{T} \tau-F_{8}$

Here $\boldsymbol{M}_{0}=\left[\begin{array}{cc}\left(m_{\mathrm{P}} \boldsymbol{I}\right)_{3 \times 3} & 0_{3 \times 3} \\ 0_{3 \times 3} & \boldsymbol{A}_{\mathrm{G}(3 \times 3)}\end{array}\right]$ is the inertia matrix of the scale model including any attached payload, $m_{\mathrm{p}}$ is the mass and $\boldsymbol{A}_{\mathrm{G}}$ is the inertia tensor about the gravity center. $\boldsymbol{M}=\operatorname{diag}\left(m_{1}, \cdots, m_{8}\right) \in \mathrm{R}^{8 \times 8}$ is the inertia matrix of the actuators $\boldsymbol{X}$ is vector of the posture of the scale model, $\quad \boldsymbol{B}=\operatorname{diag}\left(b_{1}, \cdots, b_{8}\right) \in \mathrm{R}^{8 \times 8}$ is the matrix of viscous friction of the actuators $\hat{\boldsymbol{o}}=\left(\tau_{1}, \cdots, \tau_{8}\right)^{\mathrm{T}} \in \mathrm{R}^{8}$ is vector of the torque of the actuators $\boldsymbol{F}_{\mathrm{g}}=\left(0,0, m_{\mathrm{p}} \cdot g, 0,0,0\right)^{\mathrm{T}}$ is the gravity vector of the scale model, and $\mathrm{g}=9.8\left(\mathrm{~m} / \mathrm{s}^{2}\right)$.

The total dynamic modeling is a highly coupled and redundantly restrained nonlinear system which should be decoupled and linearized.

A control law of actuator vector of motor is designed as follows:

$$
\hat{\boldsymbol{o}}=\left(J^{\mathrm{T}}\right)^{+}\left(K_{\mathrm{d}}\left(\boldsymbol{X}_{d}-\boldsymbol{X}\right)+K_{\mathrm{v}}\left(\dot{\boldsymbol{X}_{d}}-\dot{\boldsymbol{X}}\right)+F_{\mathrm{g}}\right)+v
$$

Here, $J^{T} v=0$ is satisfied. Moreover $K_{d}$ and $K_{v}$ are the different values of the control feedback gain without wind. It can be proven that the control system is stable and robust with the control law mentioned above.

It is noted that if another control law is used which can ensure the stability of the control system, the value s of dynamic derivatives calculated from the control system which will be formulated in detail in the next section will be different. A question may arise about the correctness of the method for the dynamic derivatives' calculation, but it is regarded as reasonable after a balance of the analysis of the differences of control schemes and of their robustness is given. Much more work in this aspect will be discussed in the future work. Moreover the required repeatability of the control system will be indicated and investigated using some kind of tools like robustness.

\subsection{Dynamic Derivatives' Calculation}

Taking the pitch oscillation for example (ie. $\mathrm{X}=\theta_{P}=$ $\left[\begin{array}{lllll}0 & 0 & 0 & 0 & \theta_{P}\end{array}\right]^{T}=\left[\begin{array}{lllllll}0 & 0 & 0 & 0 & \theta_{P 0} \sin \varpi \tau 0\end{array}\right]^{T}$ ), the total dynamic model without wind and with wind can be obtained. In fact, the single-DOF oscillation control of the scale model is successfully implemented using the WDPSS-8 prototype, the frequency of which is from $0 \sim 2$ $\mathrm{Hz}$ and the amplitude of which is $9 \sim 10$ degree (Liang et al. 2007). In addition, concerning the issue that the motion versus time tracks that differs by small fractions of a degree should be provided by a motion control system in order to obtain accurate measurements of the dynamic derivatives. May be a time-varying discrete control system should be built and investigated, but as the preliminary research, it is only regarded as a time-constant continuous control system. Moreover, the specifications on the path of the single-DOF oscillation of the scale model matching with wind on and wind off should be considered to an extent that target precisions are related to expected accuracies of the measured derivatives, but this issue will not be discussed here. Much more work in the aspect will be discussed in future work with the tools like the robustness of the control system.

Under the condition when the scale model has a pure pitch rotation without wind, Eq. (3) can be written as

$\left(M_{o}+J^{T} M J\right) \theta_{P}+\left(M_{o}+J^{T} M J+J^{T} B J\right) \theta_{P}=J^{T} \tau-F_{8}$

From Eq. (5), we can get $\alpha \theta_{P}+b \theta_{P}=c$, where: $a$ is the result of adding all the elements of the $5^{\text {th }}$ row of Matrix $\left(M_{o}+J^{T} M J\right), b$ is the result of adding all the elements of the $5^{\text {th }}$ row of Matrix $\left(M_{o}+J^{T} M J+J^{T} B J\right)$, $c=K_{d}\left(\theta_{P d}-\theta_{P}\right)+K_{v}\left(\theta_{P d}-\theta_{P}\right), \theta_{P}-\theta_{P d}$ and $\theta_{P d}$ are the desired pitch angle and angular velocity of the scale model. 


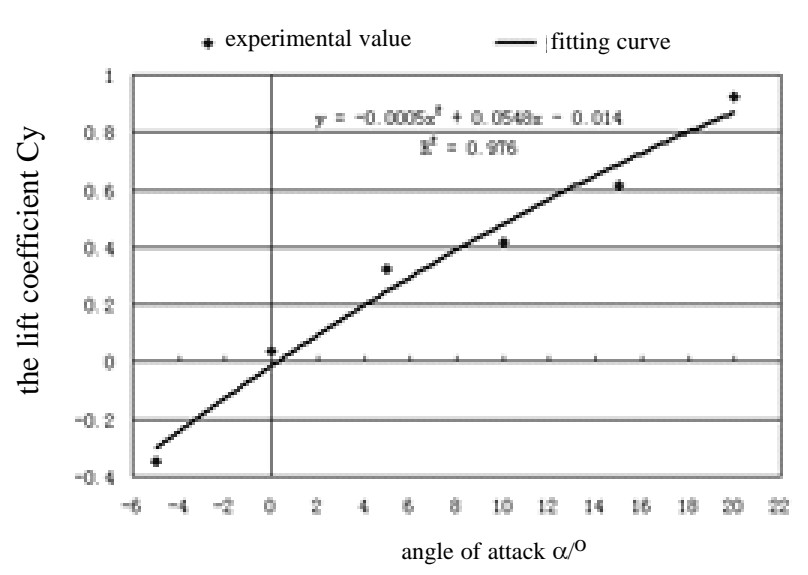

(a) the lift coefficient vs angle of attach

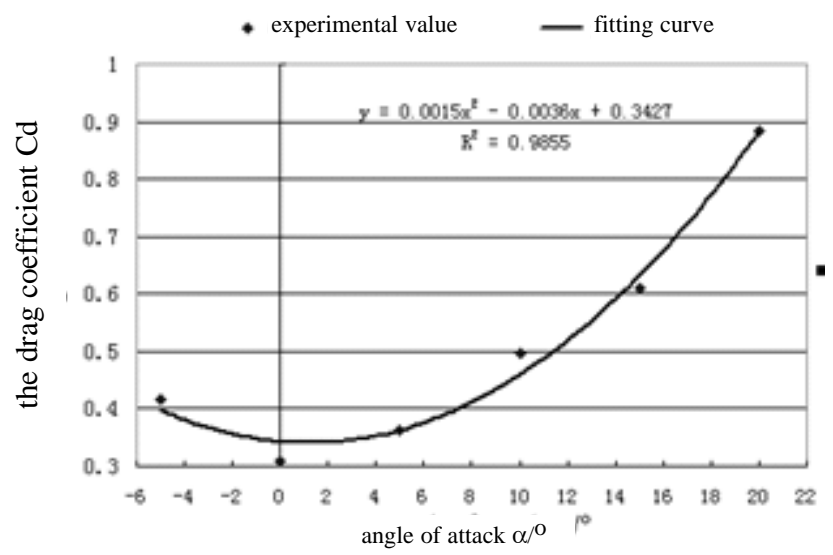

(b) the drag coefficient vs angle of attach

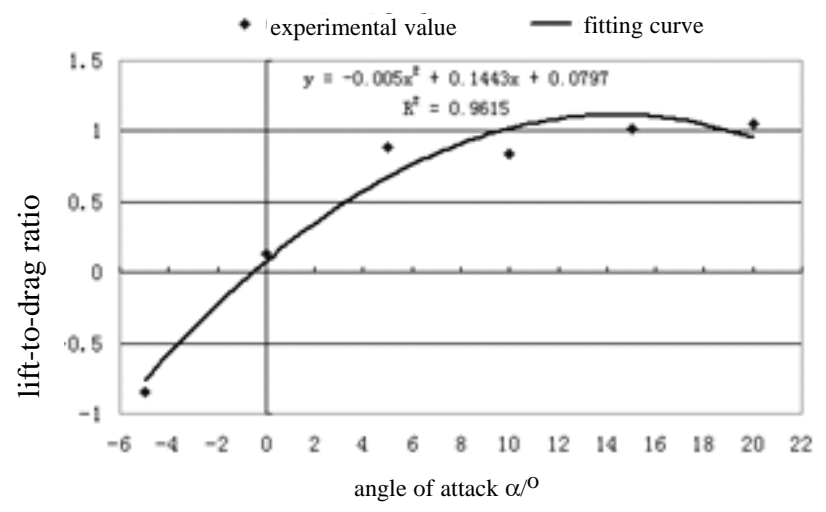

(c) lift-to-drag ratio vs angle of attach

Figure 8. Experimental curves for wind tunnel testing of WEPSS-8

$M_{y}(t)_{\text {off }}$, defined as the oscillation torque vector of the system without wind when the scale model has a pure pitch rotation, satisfies:

$$
(M) \sin (\omega t+\lambda)
$$

Under this condition when the scale model has a purepitch rotation with wind, Eq. (3) can be written as

$$
\begin{aligned}
& M_{y}^{\theta_{\mathrm{P}}} \ddot{\boldsymbol{e}}_{P}+M_{y}^{\dot{\theta}_{\mathrm{p}}} \dot{\boldsymbol{e}}_{P}+M_{y}^{\theta_{\mathrm{P}}} \grave{\boldsymbol{e}}_{P}+\left(\boldsymbol{M}_{0}+\boldsymbol{J}^{\mathrm{T}} \boldsymbol{M J}\right) \ddot{\dot{\boldsymbol{e}}}_{P}+ \\
& \left(\dot{\boldsymbol{M}}_{0}+\boldsymbol{J}^{\mathrm{T}} \boldsymbol{M} \dot{\boldsymbol{J}}+\boldsymbol{J}^{\mathrm{T}} \boldsymbol{B} \boldsymbol{J}\right) \dot{\dot{\boldsymbol{e}}}_{P}=\boldsymbol{J}^{\mathrm{T}} \hat{\boldsymbol{o}}^{\prime}-\boldsymbol{F}_{\mathrm{g}}
\end{aligned}
$$

$M_{y}(t)_{o n}$, defined as the oscillation torque vector of the system with wind when the scale model has a pure pitch rotation , satisfies

$$
\begin{aligned}
M_{y}(t)_{o n} & =(\bar{M}) \sin (\omega t+\lambda)=K_{d}^{\prime}\left(\theta_{P d}-\theta_{P}\right) \\
& +K_{v}^{\prime}\left(\dot{\theta_{P d}}-\dot{\theta_{P}}\right)
\end{aligned}
$$

From Eqs. (5) and (7), the following equation can be obtained:

$$
\begin{aligned}
M_{y}^{\ddot{\theta_{\mathrm{P}}}} \ddot{\theta_{\mathrm{P}}}+M_{y}^{\dot{\theta_{\mathrm{P}}}} \dot{\theta_{\mathrm{P}}}+M_{y}^{\theta_{\mathrm{P}}} \theta_{\mathrm{P}}= \\
\left(\left(\overline{M_{y}}\right)_{\text {on }}-\left(\bar{M}_{y}\right)_{\text {off }}\right) \sin (\omega t+\lambda)
\end{aligned}
$$

From Eqs. (6), (8) and (9), the following equation can be obtained:

$$
\begin{aligned}
&\left(\left(\overline{M_{y}}\right)_{o n}-\right.\left.\left(\bar{M}_{y}\right)_{\text {off }}\right) \sin (\omega t+\lambda)=\left(K_{d}^{\prime}-K_{d}\right) \\
&\left(\theta_{P d}-\theta_{P}\right)+\left(K_{v}^{\prime}-K_{v}\right)\left(\dot{\theta_{P d}}-\dot{\theta_{P}}\right)
\end{aligned}
$$

In fact the $5^{\text {th }}$ element of vector torque $\hat{\boldsymbol{o}}$ and $\hat{\boldsymbol{o}}^{\prime}$ of the motors, $\tau_{y}$ and $\tau_{y}^{\prime}$, can be measured by tension sensors, which can be respectively expressed by:

$$
\begin{array}{r}
\tau_{y}=a_{1 \tau_{y}} \sin \omega t+b_{1 \tau_{y}} \cos \omega t ; \tau_{y}^{\prime} \\
=a_{1 \tau_{y}^{\prime}}^{\prime} \sin \omega t+b_{1 \tau_{y}^{\prime}}^{\prime} \cos \omega t
\end{array}
$$

Hence, Eq. (9) can be expressed by:

$$
\begin{aligned}
& \left(\left(\bar{M}_{y}\right)_{\text {on }}-\left(\bar{M}_{y}\right)_{\text {off }}\right) \sin (\omega t+\lambda) \\
& \quad=J_{5}\left(a_{1 \tau_{y}^{\prime}}^{\prime}-a_{1 \tau_{y}}\right) \sin \omega t+J_{5}\left(b_{1 \tau_{y}^{\prime}}^{\prime}-b_{1 \tau_{y}}\right) \cos \omega t
\end{aligned}
$$

Here $J_{s}$ is the result of adding all the elements of the $5^{\text {th }}$ row of Matrix $J^{T}$.

Owing to the three equations: $\theta_{\mathrm{P}}=\theta_{\mathrm{P} 0} \sin \omega t$, $\dot{\theta_{\mathrm{P}}}=\omega \theta_{\mathrm{P} 0} \cos \omega t, \ddot{\theta_{\mathrm{P}}}=-\omega^{2} \theta_{\mathrm{P} 0} \sin \omega t$, Eq. (9) can be rewritten as:

$$
\begin{gathered}
M_{y}^{\theta_{\mathrm{p}}}-\omega^{2} M_{y}^{\ddot{\theta_{\mathrm{p}}}}=\frac{\left(\overline{M_{y}}\right)_{o n}-\left(\overline{M_{y}}\right)_{\text {off }}}{\theta_{P 0}} \cos \lambda=J_{5}\left(a_{1 \tau_{y}^{\prime}}^{\prime}-a_{1 \tau_{y}}\right) ; \\
M_{y}^{\dot{\theta_{\mathrm{p}}}}=\frac{\left(\overline{M_{y}}\right)_{o n}-\left(\overline{M_{y}}\right)_{o f f}}{\omega \theta_{P 0}} \sin \lambda=J_{5}\left(b_{1 \tau_{y}^{\prime}}^{\prime}-b_{1 \tau_{y}}\right)
\end{gathered}
$$

Owing to the 3 equations: $M_{y}^{\theta_{\mathrm{p}}}=M_{y}^{\alpha}+M_{y}^{\omega_{y}}$; $\left.M_{y}^{\ddot{\theta_{\mathrm{p}}}}=M_{y}^{\dot{\omega_{y}}} ; M_{y}^{\theta_{\mathrm{p}}}=M_{y}^{\alpha}\right)$, Eqs. (9) can be rewritten as:

$$
M_{y}^{\alpha}-\omega^{2} M_{y}^{\dot{\omega_{y}}}=\frac{\left(\overline{M_{y}}\right)_{o n}-\left(\overline{M_{y}}\right)_{o f f}}{\theta_{P 0}} \cos \lambda=J_{5}\left(a_{1 \tau_{y}^{\prime}}^{\prime}-a_{1 \tau_{y}}\right)
$$




$$
M_{y}^{\dot{\alpha}}+M_{y}^{\omega_{y}}=\frac{\left(\overline{M_{y}}\right)_{o n}-\left(\overline{M_{y}}\right)_{o f f}}{\omega \theta_{P 0}} \sin \lambda=J_{5}\left(b_{1 \tau_{y}^{\prime}}^{\prime}-b_{1 \tau_{y}}\right)
$$

Eqs. (11) and (12) can also be rewritten as Eq. (14) and Eq. (15) respectively, as follows:

$$
m_{y}^{\alpha}-K^{2} m_{y}^{\overline{\omega_{y}}}=\frac{\left(\overline{M_{y}}\right)_{o n}-\left(\overline{M_{y}}\right)_{\text {off }}}{\theta_{P 0} q s b_{A}} \cos \lambda=\frac{J_{5}\left(a_{1 \tau_{y}^{\prime}}^{\prime}-a_{1 \tau_{y}}\right)}{\theta_{P 0} q s b_{A}}
$$

$\left(m_{y}^{\alpha}-K^{2} m_{y}^{\overline{\omega_{y}}}\right)$ is called In-Phase Pitch Oscillatory Derivatives. $K=\frac{\omega b_{A}}{V}$ is called reduced frequency, $b_{A}$ is called mean aerodynamic chord length. $V$ is called free-stream airflow velocity.

$$
m_{y}^{\dot{\alpha}}+m_{y}^{\omega_{y}}=\frac{\left(\overline{M_{y}}\right)_{o n}-\left(\overline{M_{y}}\right)_{o f f}}{\theta_{P 0} q s b_{A} K} \sin \lambda=\frac{J_{5}\left(b_{1 \tau_{y}^{\prime}}^{\prime}-b_{1 \tau_{y}}\right)}{\theta_{P 0} q s b_{A} K}
$$

$\left(m_{y}^{\alpha}+m_{y}^{\omega_{y}}\right)$ is called Out -of-Phase Pitch O scillatory

Derivatives.

In the same way, the dynamic derivatives corresponding to the other 2 single-DOF oscillation (roll oscillation and yaw oscillation) are obtained.

However, the analysis is just based on the theoretical aspects. The test platform of WDPSS-8 for the experiment of dynamic derivatives is still to be built and the precise measuring systems of the vibration angular displacement and the real torque of the motors should be designed and implemented.

\section{Conclusions and Future Work}

The following conclusions can be drawn into after the analysis of the comparision of the 3 suspension systems including the strut suspension system, cable-mounted system and wire-driven parallel suspension system.

(1) Though strut or sting suspension systems are used in most of low-speed wind tunnels, cable mounted system is also one suitable method for measuring the static derivatives of the aircraft in LSWT which allows a large supporting stiffness, small interference of the streamline flow and a high measuring precision for large attack angle, however it can not be used in measuring the dynamic derivatives.

(2) For now, the strut suspension systems and rotary balances can be used in measuring the dynamic derivatives of the aircraft in both vertical and horizontal lowspeed wind tunnels successfully, though wire-driven parallel suspension system has a great potentiality, but it is still under investigation.
(3) The wire-driven parallel suspension system has opened a new horizon for measuring the static and dynamic derivatives of aircraft in LSWT. Using the same system based on positon control and force control in robotics allows free flight of the aircraft and obtains the static and dynamic derivatives. However, the results given in this paper can only be considered as a preliminary step in establishing feasibility though the wire-driven parallel suspension system is a very interesting design, and if it is with sufficient may be developed into a rountinely practical system.

\section{Acknowledgments}

This research is sponsored by National Natural Science Foundation of China (Grant No. 50475099),the Youth Talents Creation Foundation of Fujian Province (Grant No. 2006F3083) and the Scientific Launch Foundation for the Talents of Huaqiao University (Grant No. 06BS218).

\section{References}

Bennett, R.M., Farmer, M.G., Mohr, R.L., et al, 1978, "Wind-Tunnel Technique for Determining Stability Derivatives from Cable-Mounted Models," Journal of Aircraft, Vol. 15(5), pp. 304-310.

Bernhardt, J.E., Williams, D.R., 2000, "Close-Loop Control of Forebody Flow Asymmetry," Journal of Aircraft, Vol. 37(3), pp. 491-498.

Bian, Y.Z., Li, X.R., Li, L., et al, 1999, "Development of Model Cable Mount and Strain Gauge Balance Measuring System in Low Speed Closed Wind Tunnel," Experiments and Measurements in Fluid Mechanics, Vol.13(3), pp. 85-90, [in Chinese].

Cao, Y.H. and Deng, X.Y., 1998, "Experimental Study of Aircraft Fuselage Aerodynamic Forces at High Attack Angle," Acta Aerodynamica Sinica, Vol. 16(4), pp. 394-399, [in Chinese].

Du, X.Q., Wang, X.J. and Zhou, Y., 2003, "Investigation of Support Interference in Low Speed Wind Tunnel Oscillation Tests," Experiments and Measurements in Fluid Mechanics, Vol.17,No.2,pp.37-40, [in Chinese].

Griffin, S.A., 1991, "Vane Support System (VSS), A New Generation Wind Tunnel Model Support System," AIAA91-0398.

http://www.onera.fr/dcsd/sacso/index.php.

Hu, L., 2008, "Research on Wire-Driven Parallel Suspension Systems for Low-Speed Wind Tunnels", Master's Thesis.

Quanzhou: Huaqiao University, October 2008. [in Chinese].

Lafourcade, P., Llibre, M., Reboulet, C., 2002, "Design of a Parallel Wire-Driven Manipulator for Wind Tunnels," Proceedings of the Workshop on Fundamental Issues and Future Directions for Parallel Mechanisms and Manipulators, Quebec City, Quebec, pp. 187-194.

Liang, B., Zheng, Y.Q. and Lin, Q., 2007, "Attitude 
Control of the Scale Model of Wire-Driven Parallel Suspension.

Systems for Low-Speed Wind Tunnels," Forum on Key Technique of Large Aircraft and Academic Annual Meeting of Chinese Society of Aeronautics and Astronautics in 2007, Shenzhen, China.

Lin, Q., Liang, B. and Zheng, Y.Q., 2008, "Control Study on Mmodel Aattitude and Oscillation by Wire-Driven Parallel Manipulator Support System for Low-Speed Wind Tunnel," Journal of Experiments in Fluid Mechanics, Vol.22(3), pp. 75-79, [in Chinese].

Lin, Q., Zheng, Y.Q. and Liu, X.W., 2006, "Modeling and Control of a Wire-Driven Parallel Support System with Large Attack Angles in Low Speed Wind Tunnels," CD Proceedings of 25th Congress of the International Council of the Aeronautical Sciences, Hamburg, Germany.

Liu, T.F., Gao, F.X. and Lv, W., 2001, "Mechanical Property Calculation of Wire-Support Structure in Wind Tunnel Experiment," Mechanics in Engineering, Vol. 23(1), pp. 46-48, [in Chinese].

Liu, X.W., Q.Y., Agyemang, B.B., Zheng, Y.Q. and Lin, Q.,2006, "Design of a Wire-Driven Parallel Suspension System for Wind Tunnel based Virtual Flight Testing," Proceedings of the 7 th International Conference on Frontiers of Design and Manufacturing, Guangzhou, China.

Liu, X.W., Zheng, Y.Q. and Lin Q., 2004, "Overview of Eire-Driven Parallel Manipulators for Aircraft Wind Tunnels," Acta Aeronautica Et Astronautica Sinica, Vol. 25(4), pp. 393-400, [in Chinese].

Roos, F.W., 2001, "Micro Blowing for High-angle-ofattack Vortex Flow Control on Fighter Aircraft," Journal of Aircraft, Vol. 38(3), pp. 454-457.

Shen, L.M., Shen, Z.H. and Huang, Y., 1988, "A Wire Type-support System for High Angle of Attack Test in Lw Speed Wind Tunnel," Experiments and Measurements in Fluid Mechanics, Vol. 12(4), pp. 1522, [in Chinese].
Sun, H.S., 1999, "The Development of 96-test System for Measuring Dynamic Derivatives at High Angle of Attack," Journal of Experiments in Fluid Mechanics, Vol. 13(1), pp. 31-37, [in Chinese].

Wang, Y.K., Huang, T. and Deng, X.Y., 2004, "The Study of Aerodynamic Characteristics of after body and effect of Cable-Mounting," Chinese Journal of Theoretical Applied Mechanics, Vol. 36(3), pp. 257264, [in Chinese].

Zhang, R.P., Wang, X.N. and Li, Z.X., et al. 2006, "Investigation of Sting Support Interference in Low Speed Wind Tunnel," Journal of Experiments in Fluid Mechanics, Vol.20(3), pp. 33-38, [in Chinese].

Zheng, Y.Q., Lin, Q. and Liu, X.W., 2007, "Initial Test of a Wire-Driven Parallel Suspension System for Low Speed Wind Tunnels," In: Proceedings of the 12th World Congress in Mechanism and Machine Science, Besancon, France, June 17-21, 2007, Vol. 5, pp. 8893.

Zheng, Y.Q., Lin, Q. and Liu, X.W., 2005, "Design Methodology of Wire-Driven Parallel Support Systems in the Low Speed Wind Tunnels and Attitude Control Scheme of the Scale Model," Acta Aeronautica Et Astronautica Sinica, Vol. 26(6), pp. 774-778, [in Chinese].

Zheng, Y.Q., 2004, "Research on Key Theoretical Issues of Wire-Driven Parallel Kinematic Manipulators and the Application to Wind Tunnel Support Systems," PhD Dissertation, Quanzhou:Huaqiao University, pp. 4-104, [in Chinese].

Zheng, Y.Q., 2006, "Feedback Linearization Control of a Wire-Driven Parallel Support System in Wind Tunnels," Proceedings of Sixth International Conference on Intelligent System Design and Applications, Jinan, Shandong, China.

Zheng, Y.Q., Lin, Q. and Liu, X.W., 2006, "Kinematic Calibration of a Wire-Driven Parallel Support System in Wind Tunnels," China Mechanical Engineering, Vol.17(6), pp. 551-554. 